\title{
Original Article \\ Hospital induced psychological distress among patient attendants at Liaquat University Hospital, Jamshoro
}

\begin{abstract}
Background: Existing literature on interpersonal affairs amid health professionals and patients has proliferated since the 1960s, highlighting the importance of health professionals of all sorts. Here, in the clinical setup of Liaquat University Hospital, we have another important relationship that is crucial to a patient's health care plan, is an adjunct to the health care professionals and contributes significantly more to the patients care. This relationship, often taken for granted by the health authorities, is that of a patient and a family member voluntarily staying with the patient at the hospital (commonly termed as attendant). Objective: This study hopes to unearth the major problems (particularly hospital induced psychological distress) faced by attendants and inquire what major complaints they have against the health care setup, health professionals and the hospital. Methodology: This cross-sectional observational study was conducted from May to July 2013. A data sample of 150 patient attendants at Liaquat University Hospital, Jamshoro was chosen by purposive sampling. The data was collected by self-administered structured questionnaire and was analyzed using SPSS v.16.0 and MS Excel. Results: 66\% of the patient attendants were males while 34\% were females. The most common attendants were sons $(22 \%)$, mothers $(17.3 \%)$ and brothers $(12 \%)$. The most common complaint of the patients was that the attendants had no proper place to stay even if their stay was necessary. The attendants complained of inadequate sleep, health worries, anxiety, depressed mood, agitation, reduced appetite and weight loss due to improper food. Conclusion: We conclude, on the basis of our findings, that an important asset is being mistreated by our healthcare system and this mistake demands to be corrected on a priority basis. Steps should be taken to solve their problems so that they may continue to play their positive role.
\end{abstract}

Patient attendants, Primary caregivers, health aides, untrained medical helpers and healthcare assistants.

\section{Introduction}

Research, academic literature on interpersonal affairs amid health professionals and patients has proliferated in the last two decades, highlighting the importance of health professionals of all sorts. Here in our clinical setup we have another important relationship that is crucial to a patient's health care plan, is an adjunct to the health care professionals and contributes significantly more to the patients care. This relationship, often taken for granted by the health authorities, is that of a patient and its attendant and family.

In 1974, Litman emphasized the family as a basic unit of healthcare (Litman; 1947), and there have been several literature reviews of the family and health. (Schmidt; 1978, Robertson; 1985 \& Campbell; 1986). Campbell concluded from his review that most studies of the family's impact on physical health consider the family as a source of social support (Campbell; 1986). Hospital admission of a relative is a stressful event that has long been ignored. Family members of patients may encounter stressors that endanger personal health and wellbeing of the family likewise. In 2000, Horn et al suggested in his study (Van Horn; 2000) that family members suffer from numerous concomitant stressors and showcase multiple behavioral responses, including altered sleeping, activity, dietary and family roles. Our national health care setup is utterly negligent towards patient attendants. This has gradually piled up a lot of problems for the attendants that are seriously debilitating their ability to tend for the patient and provide them proper care and are inducing stress in themselves, risking their $\&$ the patients welfare.

The stress induced in the patients' attendant and family, can ultimately affect the patients' health. This affect however remains an unexplored and a substantially neglected area of empirical research, more so in developing than developed countries. But, in 1992, in a cross sectional study Parkerson et al demonstrated that family stress was a statistically significant predictor of function in terms of physical, mental and social health; self-esteem, anxiety; and depression (Parkerson; 1992).

A recent study measured the predictive effects of social stress and support but did not focus specifically on family stress and support. McKay et al, used the social readjustment rating scale of Holmes and Rahe to measure stress and found that patients with higher life stresses had a 1.6 to 1.8 times higher risk of nine or more days of illness and that social support buffered the effect of stress. (McKay; 1985)
It is known that in sickness, humans are more vulnerable and are easily affected by depressive stimulants. While tending to a sick patient, the attendants too are prone to develop depression. In the present study it is hypothesized that conditions at Liaquat University Hospital (LUH) are worse than other healthcare centers reported in research literature and the depressive stimulants and triggers too exist in greater abundance at LUH. We thus believe that the consequent stress and depressive symptoms in the patient's attendants to be higher than elsewhere quoted in literature.

\section{Methodology}

A cross-sectional study was conducted at Liaquat University Hospital from May to July 2014. The study was a simple observational study in design and data was collected from 150 attendants during daily visiting hours (using the Hamilton Depression Rating Scale (HDRS) along with a section of the Depression, Anxiety and Stress Scale) after taking verbal informed consent. The questions were presented to the respondents in their local languages (Urdu and Sindhi). Selection of participants was done by purposive sampling. $66 \%$ of the sample comprised of males while $34 \%$ were females. The data collected was analyzed using SPSS v.16.0 and Microsoft Excel 2013.

\section{Results}

All attendants were immediate or nearby family members. The most common attendants were sons $(22 \%)$, mothers $(17.3 \%)$ and brothers (12\%).Among the $66 \%$ were males while only $34 \%$ were females.

Relation of Attendant With Patient

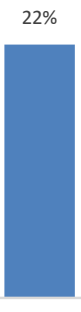

Son

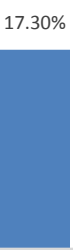

Mother
$12 \%$

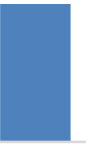

Brother 
Figure 1: Sons most commonly served as attendants followed by mothers and then brothers. The fact that 2 of the top three related attendants slots belonged to males shows that males majorly serve as the support for the sick.

The attendants complained of depressed mood, agitation and anxiety. Such self-professed depressive symptoms were unearthed by a detailed evaluation of their responses recorded by the Hamilton Depression Rating Scale and Depression, Anxiety and Stress Scale. The figure 2 below shows the extent to which these symptoms were experienced.

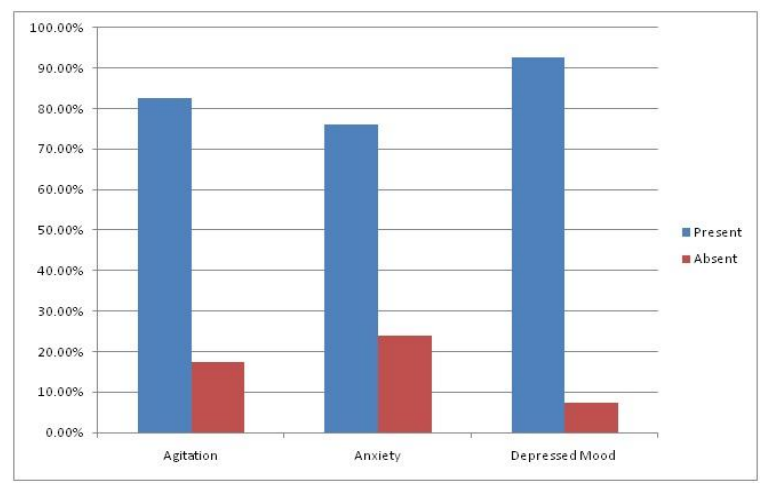

Figure 2: Depression was the most prevalent, 92.7 percent of the sample experienced depressed mood. 76 percent of the sample suffered from anxiety and a total of 82.7 admitted to feeling agitated.

A large proportion of the patient attendants reported generalized body ache ( 75.3 percent) and health related worries ( 56 percent) due to unhygienic environment as shown below. (Fig 3)

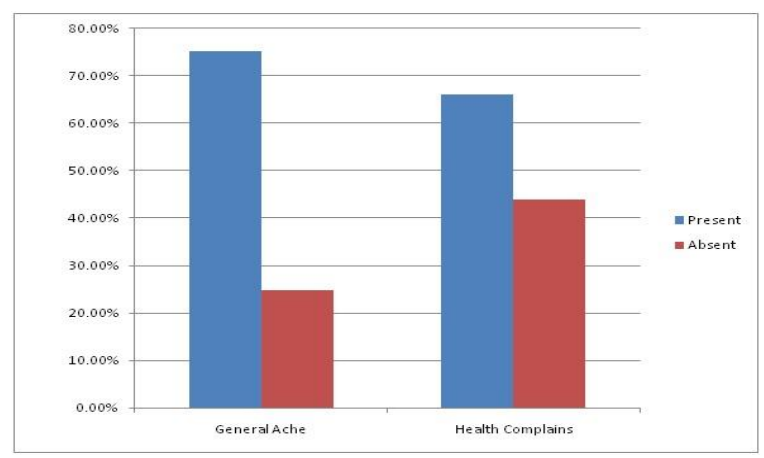

Figure 3: Somatization is a phenomenon often experienced by depressed individuals. The attendants allegedly felt a similar symptom i.e. generalized body ache unrelated to their medical condition. The poor hygiene conditions, contagious environment and psychological effect of witnessing fellow patient's suffering further aggravated the health worries.

Uncomfortable conditions, sub-standard food and unhygienic environment for eating contributed to a reduced appetite among patient attendants. Prolonged stay at the hospital and persistently low appetite often leads to loss in body weight. Figure 4 below shows the extent to which loss in appetite and weight are experienced by the sample.

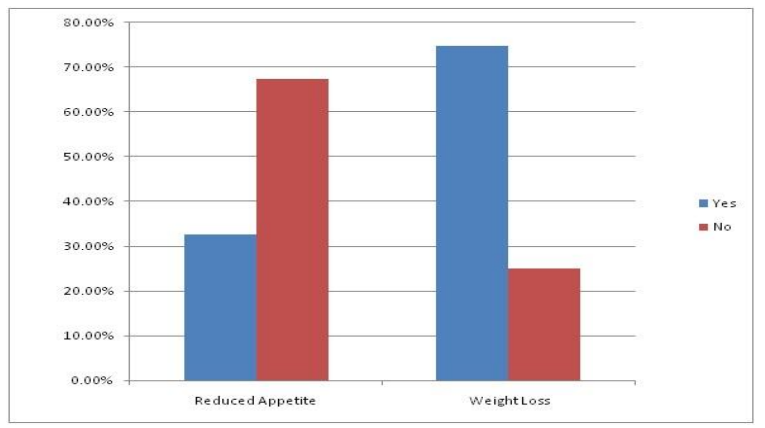

Figure 4: 32.7 percent of the reported to have experienced a loss of appetite. And as a potential consequence a total of 75.3 percent of the sample showed marked weight loss. In addition to the loss of appetite, lack of availability of good quality nutritious food is also believed to cause weight loss.

\section{Discussion}

Scarcely ever does literature offer much insight into hospital induced stress and any insight if available holds limited value since they seldom gauge stress with any amount of legitimacy and reliability. An example by Goldberg and Hillier is the most cited British study and it is confined only to the stress induced in trained health professionals. It does not take into regard, important adjuncts, such as patient attendants. (Goldberg; 1979)

Sadly, no specific scales have been designed to measure hospital induced stress. The scales under use in most of the literature have no normal population values but there exist accepted baseline values which have put the validity of inferred results in literature in doubt. (Bramely; 1988)

A current study has gauged levels of stress in health professionals and suggests that stress has risen (Sutherland; 1992). Furthermore, the study shows that nearly a third of the respondents were suffering from depression and stress (Goldberg; 1979 \& Firth; 1986). The British Medical Association recently printed a document on stress within the hospital premises (BMA: 1992). Although broad, it contains no information or references pertaining to gauged levels of stress or psychological symptoms in patient's attendants or visitors. The patient attendants by and large live and work in similar circumstances or probably worse. There is a dire need of supporting literature to adequately highlight this cause in front of the health authorities, medical community and the general public if we are to move towards solving this issue.

Patient attendants are an adjunct to the hospital staff at Liaquat University Hospital and work just as much as the hospital staff to ensure proper care of the patient. Their strenuous role thus exposes them to the same or possibly more occupational stress than the nurses, paramedics and doctors themselves even though their abilities are less (Hart; 1999). The attendants live in shabby conditions, cater to the patients' needs at all times and receive no appreciation from the health care system. They are thus plagued with stress-related disorders, including psychological disorders (e.g., depression, anxiety and post-traumatic stress disorder) and other types of emotional tension (e.g., fatigue and dissatisfaction) and maladaptive behaviors (e.g., aggression, substance abuse). This onslaught may compromise the attendants' ability to care for the patient and ultimately lead to bad patient health. This stress is also associated with various biological reactions that may ultimately result in deteriorated health conditions among the attendants themselves, such as cardiovascular disease (NIOSH; 2007).

However, differences in individual characteristics such as personality and coping skills can be very important in predicting whether and to what extent hospital conditions will lead to stress 
since what induces stress in one person may not be problematic for another. The influence of personality on the way people cope with stressful situations has been discussed many times. It has even been discussed whether stress-coping styles and personality traits could be confused (Leonardo; 2010). While the significance of individual differences cannot be set aside, scientific evidence proposes that certain conditions are almost unanimously felt as stressful. Such evidence is suggestive of the need for a heightened focus on hospital conditions as the key source of stress (Northwestern National Life Insurance Company; 1991).

Another factor triggering depression particularly among females is the ethical barrier that the society has imposed upon women. The barriers are more firm in the minds of the society and women in particular. The presence of difficulties that women cannot cope with as effectively as men as long as these barriers exist (such as sleeping in uncomfortable and awkward places, constant hospital demands to fetch medicine from different stores and aiding the patients to the public washrooms) may trigger psychological distress. We attribute these difficulties to the fact that females consequently perform the role of an attendant less often ( $34 \%$ of the sample).

\section{Conclusion}

On the basis of our result, we conclude that patient attendants experience a variety of difficulties in the hospital and complain of the ways that they are affected by them. Hospital induced psychological distress are among the leading complains and steps need to be taken to counter this problem and ensure a trouble free hospital experience for the attendants. It is the least that hospitals should aspire to do in return for the selfless services the attendants provide. This topic also requires further exploration and investigation using control groups to determine major stressors.

\section{Conflict of Interest}

All the authors disclosed that there is no Conflict of interest associated in the preparation of this article

\section{Acknowledgment}

We would like to acknowledge the intellectual assistance and technical support provided by LUMHS Research Forum and Mr. Muhammad Muneeb.

\section{References}

- Bramley PN, Easton AME, Morley S, Snaith RP. (1988). The differentiation of anxiety and depression by rating scales. Act Psychiatr Scand, 77, 133-8.

- British Medical Association Stress and the medical profession. (1992) London: BMA.

- Campbell TL. (1986). Family's Impact on Health: A critical review and annotated bibliography. Washington DC: Superintendant of documents. National Institute of Nental Health Series. Department of Health and Human Services publication. (ADM) 86-1461.

- Firth J. (1986). Levels and sources of stress in medical students. BMJ, 292, 1177-80.

- Goldberg DP, Hillier VF. (1979). A scaled version of the general health questionnaire. Psychol Med, 9, 139-45.

- Hart, P.M. (1999). Predicting employee life satisfaction: a coherent model of personality, work and non-work experiences, and domain satisfactions. Journal of Applied Psychology, 84, 564-584.

- Litman TJ. (1947). The family as a basic unit of health and medical care; a social-behavioral overview. Soc Sci Med, 8,495519.

- Leandro PG, Castillo MD. (2010). Coping with stress and its relationship with personality dimensions, anxiety, and depression. Procedia - Social and Behavioral Sciences, 5, 15621573
- McKay DA, Blake RL, Colwill JM et al. (1985). Social support and stress as predictors of illness. J Fam Pract, 20, 575-581.

- Northwestern National Life Insurance Company [1991]. Employee burnout: America's newest epidemic. Minneapolis, MN: Northwestern National Life Insurance Company.

- "NIOSH Work Organization and Stress Related Disorders". United States National Institute for Occupational Safety and Health. Retrieved 2007-12-01.

- Parkerson GR Jr, Broadhead WD. (1992). Validation of the Duke social support and stress scale using the duke health profile. Clin Epidemiol, 45, 1303-1313

- Robertson DL, Stewart TJ. (1985). Families and health: A review of clinical and research issues for primary care. Fam Pract Res J, 4, 128-151.

- Schmidt DD. (1978). The family as a unit of medical care. J Fam Pract, 7, 303-313.

- Sutherland VJ, Cooper CL. (1992). Job stress, satisfaction, and mental health among general practitioners before and after introduction of new contract. BMJ, 304, 1545-8.

- Van Horn, E., \& Tesh, A. (2000). The effect of critical care hospitalization on family members: stress and responses. Dimensions of Critical Care Nursing, 19(4), 40-hyhen. 